\title{
Foreword to the Special Focus on Advances in Symbolic and Numeric Computation
}

\author{
Amélia Loja - José Alberto Rodrigues • \\ Ana C. Conceição
}

Published online: 3 May 2016

C) Springer International Publishing 2016

This special focus of Mathematics in Computer Science is connected to SYMCOMP 2015, the 2nd International Conference on Numerical and Symbolic Computation, which was held on March 26-27, 2015, in University of Algarve, Portugal. This ECCOMAS Thematic Conference was organised jointly by Mechanical Engineering Institute and University of Algarve, under the auspice of Portuguese Society of Theoretical, Applied and Computational Mechanics. The special focus contains five contributions related to different scientific areas, combining symbolic computation applications with validated numerical methods.

Both numerical and symbolic computations are recognised as central tools in numerous fields of science and technology. Scientific modelling would fall well short of the mark if it attempted to describe problems and predicting outcomes without numerical and symbolic computation. Thus, not only broad understanding and critical appreciation of mathematical models are vital components in the training of science people, but also is the dissemination of this knowledge and its results when applied to real application problems.

It is therefore with this perspective that the five papers included in this special focus were selected. These papers propose non-trivial approaches to solve concrete problems with guaranteed results that enable purely exact methods to become faster and purely numerical methods to become more powerful.

\footnotetext{
A. Loja $(\varangle)$

ISEL, Instituto Superior de Engenharia de Lisboa, Av. Conselheiro Emídio Navarro, 1, 1959-007 Lisbon, Portugal e-mail: amelialoja@dem.isel.ipl.pt
}

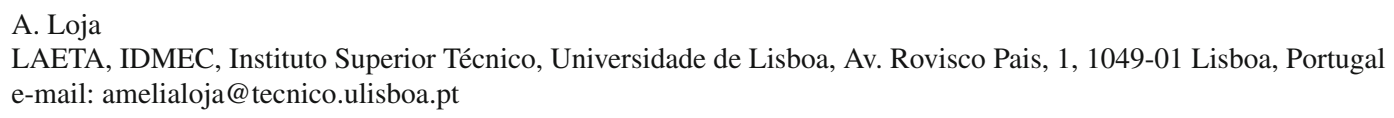

A. Loja LAETA, IDMEC, Instituto Superior Técnico, Universidade de Lisboa, Av. Rovisco Pais, 1, 1049-01 Lisboa, Portugal e-mail: amelialoja@tecnico.ulisboa.pt

\section{J. A. Rodrigues}

ISEL - Instituto Superior de Engenharia de Lisboa, Instituto Politécnico de Lisboa, Lisbon, Portugal e-mail: jrodrigues@adm.isel.pt

\section{J. A. Rodrigues}

CMAT (Centre of Mathematics) Universidade do Minho, Braga, Portugal

\section{A. C. Conceição}

CEAFEL (Center for Functional Analysis, Linear Structures and Applications), Departamento de Matemática, Faculdade de Ciências e Tecnologia, Universidade do Algarve, Faro, Portugal

e-mail: aicdoisg@gmail.com; aconcei@ualg.pt 
The main contents of these papers are summarized below.

\section{Towards a Lanczos' Tau-method toolkit for differential problems. M. Trindade, J. Matos and P. B. Vasconcelos}

The first paper of the special focus deals with building a numerical software library based on the Tau-method to approximate solutions for linear differential problems using MATLAB ${ }^{\circledR}$. A new iterative Tau-method scheme based on LU factorization and providing error estimation is proposed, serving also as available preconditioning techniques to work with Krylov iterative methods in order to ensure efficiency. This new approach can be very effective as illustrated through numerical examples.

\section{Dynamics and optimal control of Ebola transmission. Amira Rachah and Delfim F. M. Torres}

This paper presents a study on mathematical descriptions of the spread of Ebola virus using the SusceptibleExposed-Infective-Recovered model and optimal strategies for Ebola control. The virus propagation control and the prediction of the impact of vaccine programmes are investigated by considering different strategies of the spread of Ebola optimal control, namely: control infection by vaccination; minimize exposed and infected; reduce Ebola infection by vaccination and educational campaigns.

Three-dimensional computational analysis of stress state transition in through-cracked plates. Rúben Maia, Ricardo Branco, F. V. Antunes, M. C. Oliveira and Andrei Kotousov

This paper presents research results on the extent of surface regions in through-cracked planar geometries subjected to cyclic loading. A linear-elastic and an elasto-plastic model were developed to characterise the stress triaxiality state along the crack front. The extent of surface regions was evaluated using h-curves applying a newly proposed criterion. A linear relation between the stable value of the extent of surface regions and the maximum stress intensity factor was also established.

On contractions of Lie algebras. J. M. Escobar, J. Núñez and P. Pérez-Fernández

In this paper, a study on the application of the invariant functions of Lie algebras, $\psi$ and $\varphi$, to contractions of some lower-dimensional Lie algebras, is carried out. This study stands as a relevant topic, as it is known that the application of invariant functions can be found in many fields of pure and applied mathematics, physics, engineering and science in general.

Symbolic computation applied to the study of the kernel of a singular integral operator with non-Carleman shift and conjugation. Ana C. Conceição, Rui C. Marreiros and José C. Pereira

This final paper makes use of Mathematica ${ }^{\circledR}$ capabilities to implement the extensive symbolic and numerical calculations present in the analytical algorithms, devoted to the exploitation of the dimension of some classes of singular integral operators' kernel. The implementation of these methods on a computer provides new and powerful tools that make the results of lengthy and intricate calculations available in a simple way to researchers.

As guest editors, we are pleased to have been involved in the construction of this special focus and in making it now available to the community. We hope that the papers presented here may provide an illustrative picture of the diversity of applications of numerical and symbolic computation as well as different techniques used in the modelling and analysis of various problems in science and technology.

We thank the authors for having submitted their papers for publication in the special focus and the anonymous referees for having kindly volunteered their time to review the papers. Finally, we wish to thank the Editor-in-Chief, Professor Dongming Wang, and the editorial staff for their help and support. 